\title{
Effects of light or dark phase testing on behavioural and cognitive performance in DBA mice
}

\author{
Angelika Roedel ${ }^{1}$, Corinna Storch ${ }^{1}$, Florian Holsboer ${ }^{1}$ and Frauke Ohl ${ }^{1,2}$ \\ ${ }^{1}$ Behavioural Phenotyping, Max Planck Institute of Psychiatry, Kraepelinstr. 2, D-80804 Munich, \\ Germany; ${ }^{2}$ Department of Animals, Science \& Society, Faculty of Veterinary Medicine, University of \\ Utrecht, PO Box 80.166, 3508 TD Utrecht, The Netherlands
}

\section{Summary}

Behavioural experiments in mice are often carried out during the resting phase of these nocturnal animals. Ignoring the fact that mice are more active during the dark period, results from resting-phase testing has also been used to characterize these animals. Since the influence of the light/dark cycle on testing is likely to be a relevant factor for the analysis of behavioural results, the aim of this study was to evaluate the effects of the relative time of the day as well as light conditions during testing on behavioural and cognitive performance in inbred mice. Naïve DBA/2N (DBA) mice were tested in the modified hole board (mHB) either during the dark phase under red light or during the light phase under white light. Different behavioural dimensions and cognitive functions were evaluated in parallel. Depending on the testing conditions, the results showed significant differences in behavioural activity, with DBA mice being less inhibited during dark phase. The same experimental group made fewer memory errors in a visuo-spatial task and showed a faster habituation compared with the group tested during the dark phase. From the results we conclude that testing during the light phase induces a pronounced behavioural inhibition as well as a cognitive disruption in DBA mice, which should be taken into account when cognitively testing these animals.

Keywords Inbred mice; DBA/2; dark/light cycle; behaviour; cognition

Physiology and behaviour are fundamentally modulated by the light/dark cycle (Albrecht \& Oster 2001). This rhythm profoundly influences body temperature (Weinert \& Waterhouse 1998), endocrine rhythms (Ruiter et al. 2003), food intake (Stephan \& Zucker 1972, Campuzano et al. 1999), activity (Kamei et al. 1998, Takahashi \& Turek 2000, Weinert et al. 2002) and social behaviour (Stephan \& Zucker 1972).

Correspondence: Professor Dr Frauke Ohl, Chair of Laboratory Animal Science, Department of Animals, Science \& Society, Faculty of Veterinary Medicine, University Utrecht, PO Box 80.166, 3508 TD Utrecht, The Netherlands. Email: f.ohl@vet.uu.nl
Disruption of the light/dark cycle is known to cause severe physiological and behavioural dysfunction (Van der Hoofdakker \& Beersma 1988, Barbini et al. 1996, Leamann 1999). When performing experiments in animals, the relative daytime of testing (i.e. light or dark phase, respectively) as well as the light conditions used (i.e. red light or white light) should be carefully considered since both are likely to represent confounding factors for the experimental results.

A number of studies have been performed on laboratory rodents to investigate the possible effects of light conditions and time 
of testing in relation to the respective activity cycle on experimental behaviour, and varying results have been obtained. Rats in the forced-swim test were less stressed during the active phase of their circadian cycle (Kelliher et al. 2000). A comparison of various inbred mouse strains revealed differences in the circadian rhythm of locomotor activity between strains (Kopp 2001): individuals from the C57BL/6J and BALB/cJ strains showed a clear-cut decline of their locomotor activity in the second part of the night. Activity in $\mathrm{C} 3 \mathrm{H} / \mathrm{HeJ}$ and CBA/J mice declined progressively, reaching daytime values at the end of the night. All mouse strains tested displayed their main activity peak at the beginning of the dark phase.

Based on the fact that general activity levels are likely to confound behavioural measurement, it seems obvious that a defined and constant time point of testing in relation to the animals' activity cycle is of fundamental importance to obtain reliable results in behavioural studies. Nevertheless, experimental protocols show considerable variances in terms of the time point of testing. It should be considered whether or not the influence of the light/dark cycle might explain contradictory findings reported for behavioural testing in mice. Although there are only a few studies conceptualized to investigate this question (Kopp et al. 1998, Valentinuzzi et al. 2000, Johns \& King 2001, Bertoglio \& Carobrez 2002, Chaudhury \& Colwell 2002), it has been demonstrated that cognitive functions, such as acquisition, recall and extinction of memory, differ depending on the time point of testing.

The present study was designed to investigate the influence of the relative time of testing and the corresponding lighting conditions as potential confounding variables in behaviour in general as well as in visuo-spatial memory processes in mice. Inbred DBA/2N (DBA) mice were tested during either the dark (i.e. activity) phase under red light conditions or during the light (i.e. resting) phase under white light conditions. The DBA strain has been studied extensively in a number of different tests and is thought to represent an interesting animal model of high anxiety (Trullas \& Skolnick 1993, Crawley et al. 1997, Griebel et al. 1997, Ohl et al. 2003). Animals of this strain have also been reported to have distinct cognitive dysfunction in performing spatial memory tests (Ammasari-Teule et al. 1995, Crawley et al. 1997, Parmigiani et al. 1999, Cahib et al. 2002). It is of note that the same strain was shown to perform well in a visuo-spatial cognitive task when tested during their activity phase (Ohl et al. 2003). Therefore, the DBA mouse strain represents an interesting strain for investigating more systematically the effects of light or dark phase testing on behaviour and cognitive performance.

For behavioural and cognitive testing, the modified hole board (mHB) test was used, which allows the evaluation of a variety of behavioural dimensions (Ohl et al. 2001a,b). The mHB has been designed to allow for a detailed behavioural analysis based on one single paradigm and represents a test for unconditioned behaviour, at the same time minimizing the presence of test-induced aversive stimuli, such as aversive lighting conditions or forced exposition to aversive areas. In addition to the evaluation of a variety of behavioural patterns such as exploration, locomotion, avoidance behaviour, risk assessment and feeding, the $\mathrm{mHB}$ can be used to analyse cognitive processes in parallel (Ohl et al. 2002, 2003), thus, offering an ideal tool for evaluating environmental effects on behaviour and cognition in mice.

\section{Methods}

\section{Animals}

All studies were performed with naïve, 10week-old male DBA mice $(n=22)$, obtained from Charles River Laboratories (Sulzfeld, Germany). The animals were kept under standard laboratory conditions $\left(22^{\circ} \mathrm{C}, 60 \%\right.$ humidity with standard mice chow, Altromin 1314, Lage, Germany, and water available ad libitum) in the animal facilities of the Max Planck Institute of Psychiatry. The mice were randomly assigned to two 
experimental groups and were housed in the respective test rooms for two weeks before the start of the experiment under the following conditions: for testing during their active period (DBA-a, $n=11$ ), lights were on at 00:00 $\mathrm{h}$ until 12:00 $\mathrm{h}$ and for testing during the resting phase (DBA-r, $n=11$ ), lights were on at 06:00 h until 18:00 h. To avoid intermale aggression all mice were housed individually in standard Plexiglas cages under conventional conditions $(26 \times 20 \times 14 \mathrm{~cm})$. The test set-up was located behind a partition to prevent waiting animals from observing the testing procedure. From previous studies there was no evidence for testing order effects on behavioural data.

The animal studies were conducted in accordance with the guidelines set by the National Institutes of Health, USA, and the Guide for the Care and Use of Laboratory Animals of the Government of Bavaria, Germany.

\section{Modified hole board}

The $\mathrm{mHB}$ represents a combination of a hole board and an open field. The set-up used for initial testing consisted of a box $(100 \times 50 \times 40 \mathrm{~cm})$ with a hole board $(40 \times 25 \times 1 \mathrm{~cm})$ positioned in the centre of this box. The set-up was of opaque grey PVC with 23 holes staggered in three lines (Ohl et al. 1998, 2002). The holes were covered with movable lids, which were made of the same material as the board. The board was placed in the middle of a PVC box representing the central area of an open field. The area surrounding the board was divided into 12 squares of equal size by grey lines.

For cognitive testing, the experimental box was reduced in size to $50 \times 50 \mathrm{~cm}$ and a smaller board was used. On the board, 10 cylinders $(3.0 \times 3.0 \mathrm{~cm})$ were placed in two lines. Each cylinder contained a piece of almond, fixed beneath a grid, which could not be removed by the mice. In addition, the bottom of each cylinder was scented with vanilla (vanilla flavour dissolved in water $0.02 \%$; Micro-Plus, Stadtoldendorf,

Germany), as pilot studies showed that mice were attracted by this flavour. For each visuo-spatial task, three randomly selected cylinders were marked with a white (i.e. highly contrasting to the grey background) tape and also baited with a removable piece of almond as food reward. This design enabled the performance of a visuo-spatial task by changing the positions of the baited cylinders and, in doing so, to investigate flexible cognitive processes (Ohl et al. 1998). For spatial tasks, the marked cylinders were removed and replaced with unmarked cylinders, while the sequences of the baited cylinders remained unchanged. As the character of this task was identical to the mHB task, the cylinders are referred to as holes in the following.

\section{Testing schedule Testing took place} between 13:00 and 17:00 h throughout. Depending on the respective light/dark cycle in the housing/testing room, this time frame represented either the first part of the dark phase, when animals are most active or the second part of the light phase of the animals. The latter was chosen to allow animals to rest several hours before being tested. During the dark phase, the trials were executed under red light, with two spots illuminating the mHB (5 Lux, Spot R63, General Electric, $40 \mathrm{~W}, \mathrm{E} 27, \varnothing 63 \mathrm{~mm})$. When tested during the light phase, the mHB was illuminated by 70 Lux.

For two days before the experiment, each animal received one piece of almond (about $0.05 \mathrm{~g}$ ) daily for habituation, as almonds were used as familiar food in the initial $\mathrm{mHB}$ test and as food reward in the visuo-spatial test. After two weeks of habituation in the testing room, naïve mice were tested using the $\mathrm{mHB}$. For initial testing, a piece of almond (familiar food) and a food pellet (unfamiliar food; Precision Pellets F0021-J; Bioserv, Holton Industries, Frenchtown, USA) were placed at $2 \mathrm{~cm}$ distance from each other in each of the two corners close to the starting point. All individuals were successively placed into the $\mathrm{mHB}$ for $5 \mathrm{~min}$. Each animal was transferred directly from its home cage to the experimental box and placed at the start position in always the same corner of the compartment. Two days after the initial behavioural testing, cognitive testing was 
started with the same animals for nine days. Each mouse performed four trials daily, except for the last day when only three trials were run. For each of the animals, the intertrial interval was about $30 \mathrm{~min}$. After trial 20, a break of two days was inserted. For the 12th trial, the sequence of baited holes was randomly changed. For the 20th trial, the marked holes were replaced with unmarked holes (spatial trial). For the 25th trial, the sequence of the baited and marked holes was changed again. During the last trial (trial 35), the sequence of the marked holes remained unchanged but no food reward was presented. All tests were videotaped and directly monitored by a trained observer. The video set-up used was situated in the testing room. It was regularly turned on during the habituation period although no ultrasound was detected.

\section{Behavioural parameters}

The following parameters were measured and assigned to different behavioural categories according to previous studies (Ohl et al. 2001a,b, 2002):

(1) Indicating directed exploration: the latency to the first hole visit (latency hole), the total number of holes visited (holes visited), and the total time each mouse spent performing the trials (i.e. visiting all three baited holes) (time trial) (a hole was counted as visited when the mouse dipped the nose below the rim of the cylinder).

(2) Indicating anxiety/avoidance of the unprotected area: the latency to the first board entry (latency board), number of board entries, the percentage of time spent on the board ( $\%$ time board) (a board entry was scored when the animals' head and both forepaws were on the board) and risk-assessment (stretched attends).

(3) Indicating arousal or de-arousal: the percentage of time spent self-grooming (\% grooming) and the latency to selfgroom (latency grooming).

(4) Indicating locomotor activity: the number of line crossings (line crossings) (a line crossing was scored when the animal had passed a line with all 4 paws).

(5) Indicating general exploration: the number of rearings (rearings).

(6) Indicating food intake inhibition: the latency to explore familiar/unfamiliar food (exploration familiar/unfamiliar food).

\section{Cognitive parameters}

The number of holes visited was counted until the animal found each of the three food rewards or for a maximum time of $5 \mathrm{~min}$. A hole was counted as visited when the mouse dipped the nose below the rim of the cylinder. The visit of a non-baited hole was defined as wrong choice. This was regarded as equivalent to a long-term memory error, because the recall of previously learned information, such as the association between a visual mark and the food reward, is necessary to avoid wrong choices. Revisits of non-baited holes were also counted as wrong choices since animals displayed no inclination to learn the respective positions of the non-baited holes. Revisits of baited holes were defined as repeated choice, indicating a working memory error. The number of omission errors was also analysed, representing the number of omitted marked and baited holes during one trial.

\section{Statistical analysis}

Data of initial testing were analysed using a one-way analysis of variance (ANOVA, STATISTICA, Statsoft, USA) with 'phase' as factor. Data of cognitive testing were analysed using a two-way repeated measured ANOVA with 'phase' and 'trial' as main factors. Further post hoc analysis was done by the Fisher's least significant difference (LSD) test. Not normally distributed data were analysed by means of a Mann-Whitney $U$-test (between experimental groups) or Wilcoxon matched pairs test (between trials). Data were considered significant at $P<0.05$ and were expressed as group mean \pm standard error of the mean (SEM). 


\section{Results}

\section{Initial testing}

The results of the initial testing revealed a significant impact of the daytime of testing on behaviour in DBA mice. DBA mice performed significantly more stretched attends during the activity phase

(mean \pm SEM: $7.5 \pm 1.53$ (DBA-a), $2.0 \pm 0.61$ (DBA-r); $F_{(1,28)}=9.67, P<0.01$, Figure 1a). Locomotor activity (line crossings: mean \pm SEM: $119.62 \pm 4.8$ (DBA-a), $70.48 \pm 60.52$ (DBA-r); $F_{(1,28)}=13.8, P<0.05$, Figure $1 \mathrm{~b}$ ) as well as general exploration (rearings: mean \pm SEM: $41.5 \pm 3.67$ (DBA-a), $15.17 \pm 1.92$ (DBA-r); $\left(F_{(1,28)}=13.8, P<0.05\right)$, Figure 1c) was significantly higher during the activity phase when compared with the resting phase. Moreover, DBA mice showed a more pronounced inhibition of food exploration and intake during the resting phase (exploration unfamiliar food: mean \pm SEM: $2.87 \pm 0.51$ (DBA-a), $159.07 \pm 21.95$ (DBA-r); $F_{(1,28)}=8.74$, $P<0.05$, Figure $1 \mathrm{~d}$; intake unfamiliar food: mean \pm SEM: $300.0 \pm 0.0$ (DBA-a), $254.3 \pm 14.44$ (DBA-r); $F_{(1,28)}=4.59, P<0.05$, Figure 1e) compared with the situation found during the animals' activity phase, while no difference was found for the exploration of familiar food. No significant effect was found for avoidance behaviour towards the unprotected area (Figure 1f). Finally, DBA mice showed a higher defaecation rate during the resting phase (mean \pm SEM: $0.12 \pm 0.12$ (DBA-a), $0.66 \pm 0.19$ (DBA-r); $F_{(1,28)}=30.8, P<0.01$, data not shown), while no differences in terms of latency to selfgrooming was noted.

\section{Cognitive testing}

Cognitive parameters When tested during the resting phase, the animals performed a higher number of wrong choices (mean \pm SEM: $1.83 \pm 0.13$ (DBA-a), $3.14 \pm 0.25$ (DBAr); phase $\times$ trial: $F_{(34,340)}=2.64, P<0.01$, Figure 2a). The post hoc analysis indicated a significant impairment in cognitive performance especially for the first half of the experiment (trials 10,11, 13-15 and 17) and during the last trial (35) when no food reward was placed in the marked holes. In addition, a higher number of omission errors (mean \pm SEM: $0.43 \pm 0.05$ (DBA-a), $0.74 \pm 0.08$ $\left(\right.$ DBA-r); phase $\times$ trial: $F_{(34,340)}=1.90$, $P<0.01$, data not shown) was revealed for the same experimental group. Paralleling the results found for wrong choices, especially the first half of the experiment under resting phase conditions was characterized by deficits in performance.

For repeated choices, regarded as working memory errors (mean \pm SEM: $1.2 \pm 0.09$ (DBA-a), $1.31 \pm 0.14$ (DBA-r); phase $\times$ trial: $F_{(34,340)}=1.70, P<0.01$, data not shown), DBA mice showed a lower number of errors during the activity phase. The post hoc analysis revealed significant differences between experimental groups for trial 4, trial 13 and trial 35. A significant increase of repeated choices was found in trials 10, 13 and 35. Regarding the parameter time trial (mean \pm SEM: $134.61 \pm 6.64$ (DBA-a), $228.56 \pm 7.63$ (DBA-r); phase $\times$ trial: $F_{(34,340)}=1.84, P<0.01$, Figure $\left.2 \mathrm{~b}\right)$, DBA mice needed significantly less time to visit all baited holes during the activity phase. From trial 5 onwards, they showed a significant reduction of time trial compared with trial 1 when tested during the activity phase. In contrast, the mice tested during their resting phase reached a stable cognitive performance only from trial 18 onward.

Behavioural parameters Independent of the time point of testing, DBA mice showed a clear increase in time spent on the board over time (trial: $F_{(34,340)}=3.25, P<0.01$, Figure 2c). However, when tested during the resting phase, the animals showed a higher anxiety-related behaviour during the first five trials.

In terms of general exploration (rearings), no effect of the time point of testing was found, while in both experimental groups the number of rearings decreased over time (trial: $F_{(34,340)}=3.11, P<0.01$, data not shown). In trial 5, DBA mice showed a significant decrease of rearings compared with the prior trial.

The directed exploration (number of holes visited) significantly differed in DBA mice 

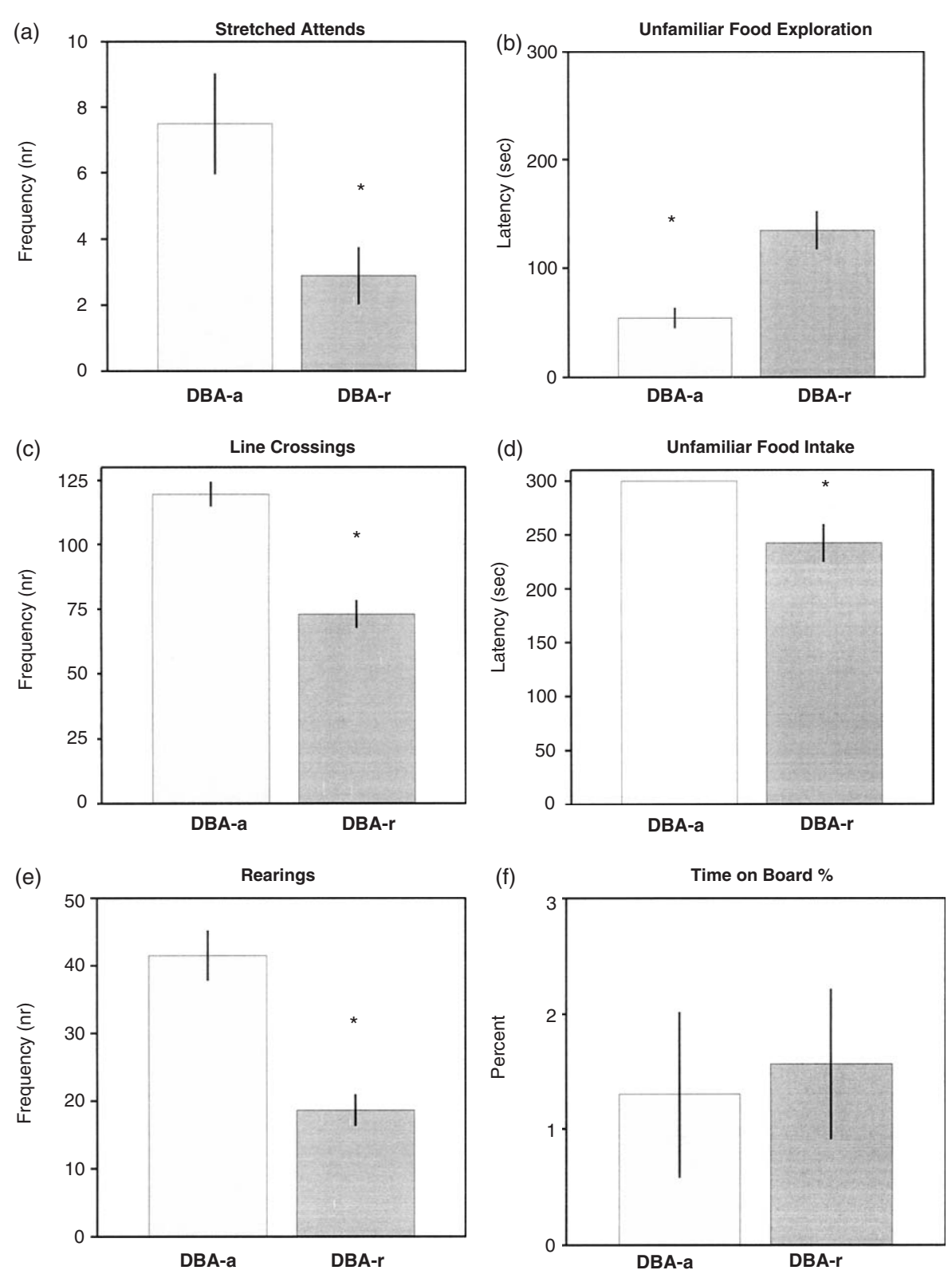

Figure 1 Results of the initial 5 min exposure to the modified hole board (mHB) test. Data are presented as mean \pm SEM. ${ }^{*} P<0.05$ between experimental groups $(n=11)$. (a) The higher number of stretched attends during activity phase (DBA-a) suggested a more active risk-assessment behaviour in DBA mice when compared with animals of the same strain tested during their resting phase (DBA-r). (c) During activity phase (DBA-a), the number of line crossings made by the animals was significantly higher than in resting-phase-tested mice (DBA-r). (e) The number of rearings either during resting (DBA-r) or activity phase (DBA-a) differed significantly. During their activity phase, DBA mice displayed an increased explorative behaviour. (b, d) The latency to explore (b) and eat (d) unfamiliar food was significantly lower in mice tested during their activity phase (DBA-a). (f) The time spent on board remained unaffected by the time of testing 
depending on the time point of testing (mean \pm SEM: $5.19 \pm 0.18$ (DBA-a), $6.06 \pm 0.39$ $\left(\right.$ DBA-r); trial $\times$ phase: $F_{(34,340)}=2.76$, $P<0.01$, data not shown). While in the beginning of the cognitive training, the mice visited more holes when tested in the activity phase (trials 3 and 4), the situation reversed in the second half of the experiment.

The interaction of trial $\times$ phase revealed a significant effect (mean \pm SEM: $33.12 \pm 3.07$ (DBA-a), $106.66 \pm 4.07$ (DBA-r); $F_{(34,340)}=$ 1.76, $P<0.01$, data not shown) for the parameter latency hole. Overall, DBA mice took more time until they visited the first hole when tested during the resting phase (trials 2, 4, 7-11, 14, 16, 20, 24 and 26).

Significant effects were found for the parameter line crossings on the interaction of trial and strain (mean \pm SEM: $36.75 \pm 2.2$ (DBA-a), 29.64 \pm 1.56 (DBA-r); $F_{(34,340)}=1.58$, $P<0.05$, data not shown). During the activity phase, DBA mice made significantly less line crossings. For the percentage of time spent on grooming, significant effects were found on the interaction of trial and phase (mean \pm SEM: $0.56 \pm 0.09$ (DBA-a), $4.76 \pm 0.35$ (DBA-r); $F_{(34,340)}=1.50, P<0.05$, data not shown), with individuals spending more time on grooming during the resting phase.

\section{Discussion}

Behavioural experiments in mice are carried out almost exclusively during the resting phase of these nocturnal animals. Ignoring the fact that these animals are more active during the dark phase, the results from light phase testing have been used to characterize rodents in general. In our study, we evaluated the effects of light or dark phase testing, respectively, on behavioural and cognitive processes in DBA mice.

Contrasting behavioural and cognitive characteristics have been described for this inbred strain: some studies present DBA mice as cognitively impaired (Schwegler et al. 1990), while others do not (ThinusBlanc et al. 1996). It is of note that different test conditions were used in these studies in terms of daytime of testing.

\section{Initial testing}

When tested during the dark phase, naïve DBA mice were more active in terms of undirected exploration (rearings, Figure 1e) as well as locomotion (line crossings, Figure 1c) compared with their behaviour during the light phase. In addition, the same animals showed a higher number of stretched attends (Figure 1a), indicating a more active risk-assessment behaviour (Rodgers et al. 1999). The stimulating effect of activity-phase testing in DBA mice was further underlined by the reduced latency to explore and eat unfamiliar food (Figures $1 \mathrm{~b}$ and d). Notably, avoidance behaviour (time on board, Figure 1f) remained unaffected by the different testing conditions. This may be explained by the high innate anxiety, which has repeatedly been described as a constant characteristic in DBA mice under various test conditions (Crawley et al. 1997, Rodgers et al. 1999, Ohl et al. 2003).

The results emphasize an inhibitory impact of testing during the light phase on behaviour in mice. Given previous findings, this effect seems to be true for rats and mice in general, since the same has been shown in selective tests for locomotor activity (Gordon et al. 1998, Kopp et al. 1998, Valentinuzzi et al. 2000, Benstaali et al. 2001) and anxiety (Bertoglio \& Carobrez 2002). Other studies in rodents could not reproduce the inhibitory effect, which was explained by methodological differences, such as the level of illumination or differences in the time of adaptation to the new light/dark cycle (Becker \& Grecksch 1996, Johns \& King 2001).

\section{Cognitive testing}

During cognitive testing, when repeatedly exposed to the test set-up, DBA mice showed a pronounced delay in habituation when tested during the light phase. When exposed to the cognitive version of the mHB for the first time (i.e. after having been initially tested 3 days before), animals tested during the dark phase already spent almost $40 \%$ of the time on the board (Figure 2c). In contrast, DBA mice tested during the light phase started the learning phase with about $20 \%$ 
time on the board and needed about 10 trials (i.e. more than 2 days) to reach the level of the dark phase group. The effect of the relative daytime of testing on cognitive performance was even more pronounced: those animals tested during the light phase needed more time (Figure $2 \mathrm{~b}$ ) to complete the test and made more errors (Figure 2a). Notably, during the light phase, DBA mice were as well more sensitive to a new challenge; this can be seen by the significant increase in error rate and 'time trial' during trial 35, when no rewards were presented with the marked holes. Overall, testing
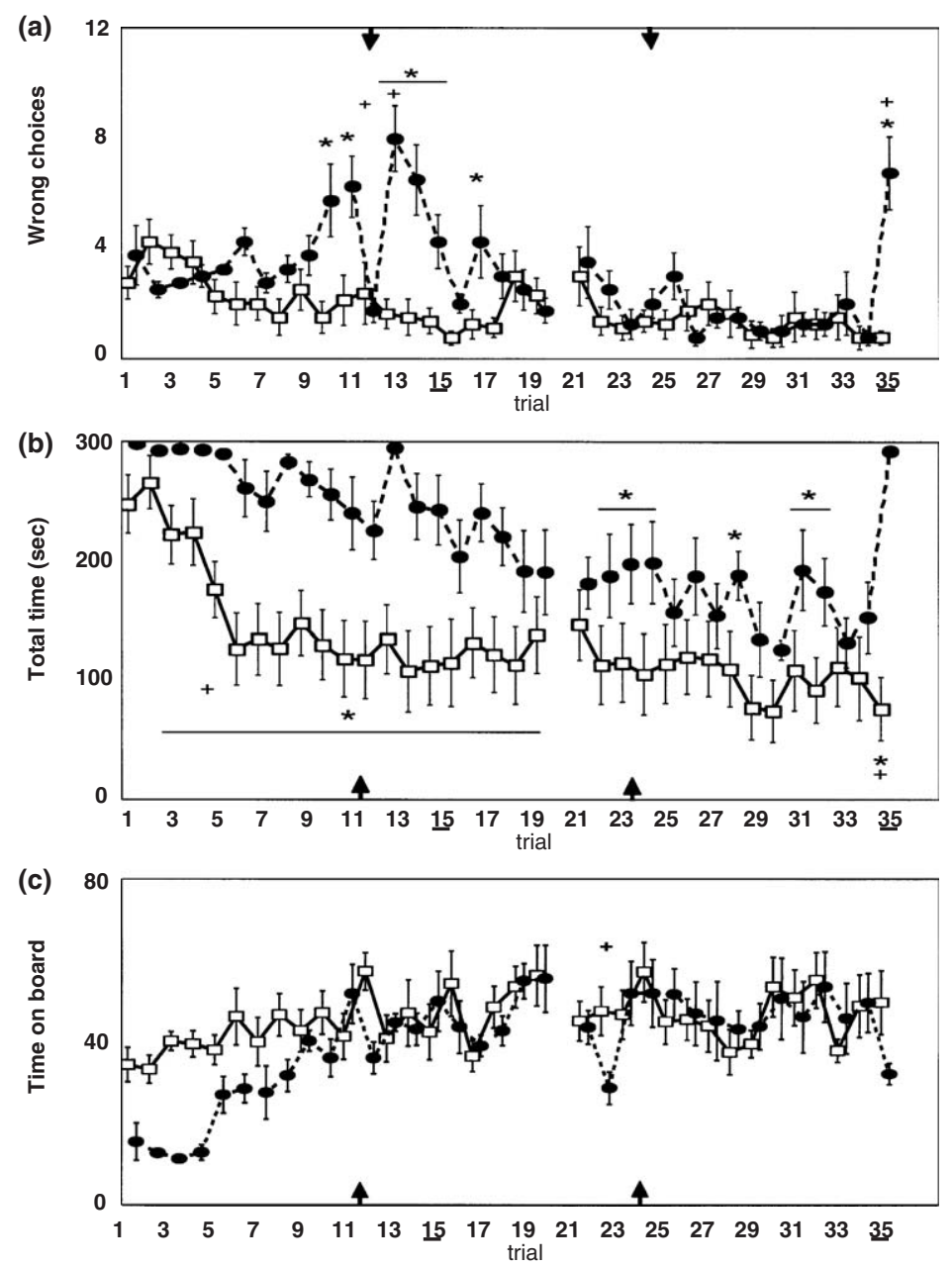

Figure 2 The number of wrong choices (a), time needed to perform the trial (b) and time spent on the board (c) in DBA mice tested during the resting phase (dotted line) and during the activity phase (solid line), respectively. When tested during the resting phase, DBA mice overall performed more wrong choices, needed more time to complete the test and spent less time on the board. The fact that differences between testing conditions were especially prominent during the first half of the experiment shows that DBA mice need longer time to habituate to the test situation when tested during their resting phase. The pronounced reaction during trial 35 indicates that resting-phase-tested animals are more vulnerable to a cognitive stressor than DBA mice tested during their activity phase. (The break of two days after trail 20 is indicated by the break of the lines. Arrows mark the introduction of a new sequence of baited and marked holes. The underlined trial 15 indicates one trial without marked holes (spatial trial). The underlined trial 35 indicates one trial where the marked holes remained unbaited.) ${ }^{*} P<0.05$ between groups. ${ }^{+} P<0.05$ versus prior trial. Data are represented as means \pm SEM 
during the light phase significantly impaired cognitive performance in DBA mice.

As mentioned above, the impaired cognitive performance in DBA mice tested during the light phase is paralleled by increased avoidance behaviour, which is indicative of anxiety. This is of note since in previous studies it has been shown that the high innate (trait) anxiety in DBA mice leads to a successful, goal-directed strategy when compared with the performance of a lowanxiety strain when tested during their activity phase (Ohl et al. 2003). In contrast, situation evoked (state) anxiety, as it is induced by resting phase testing, obviously interacts in a different way with cognitive processes. At first glance, it may be considered that the coincidence of cognitive performance and anxiety-related behaviour can be explained by the inhibitory capacity of increased anxiety (Ralph \& Mrosovsky 1992, Nguyen et al. 2000), i.e. a secondary effect on cognitive performance. This explanation, however, seems unlikely since the remarkable increase in error rates in trial 35 is paralleled by an enhanced number of hole visits, i.e. by a dis-inhibition of exploratory activity.

It also has to be considered whether or not the shift of the light/dark cycle affects the cognitive performance of mice. A light/dark reversal has been shown to produce a transient period of disruption on mice behaviour (Van den Pol et al. 1998, Weinert et a1. 2002), such as passive avoidance behaviour or emotional responses in an open field (Kopp 2001). Long-term memory processes, which in our study were assessed by means of wrong choices and omission errors, are hypothesized to be especially vulnerable to the effects of altered circadian rhythms: phase shifting of circadian rhythms are known to disrupt cognitive performance independent of its effects on non-cognitive behavioural performance (Winocur \& Hasher 1999, Devan et al. 2001, Chaudhury \& Colwell 2002). However, the time to adapt to a $12 \mathrm{~h}$ light/dark reversal has been reported to differ between inbred mouse strains ranging from four days for C57BL/6J mice to nine days for $\mathrm{C} 3 \mathrm{H} / \mathrm{He}$ mice (Kopp et al. 1998). In the study presented here, phase shifting two weeks prior to the testing had no disruptive impact on cognitive function in DBA mice. On the contrary, DBA mice made fewer long-term memory errors (wrong choices) after a fortnight of adaptation to a modified light/ dark cycle than animals that did not undergo the phase shifting. Accordingly, the number of omission errors and the time needed to perform the trial indicated a better cognitive performance in animals tested during their activity phase. Overall, the results indicate that the potentially disruptive effect of a phase shift on cognitive performance is no longer present after two weeks of habituation in DBA mice.

It should be noted that in the present experiment two factors differed between the experimental groups: relative time of testing and light conditions during testing. The $\mathrm{mHB}$ testing was performed using $70 \mathrm{Lux}$, which can be considered as non-aversive light conditions (Hascoet et al. 2001). In comparison to the light conditions used during resting phase testing (red light, 5 Lux), it cannot be excluded that the use of white light per se may be stressful to the animals, thus affecting their behavioural performance (Valentinuzzi et al. 2000, Kopp 2001, Mistlberger \& Holmes 2001). However, this study was designed to directly compare the two most frequently used test conditions, which are (i) testing during the light phase under white light conditions and (ii) testing during the dark phase under red light conditions.

It is certainly necessary to study further time points in either light or dark phase to unravel the impact of circadian rhythm on behavioural and cognitive behaviour in mice. From the results given here, we conclude that testing during the light phase induces a pronounced behavioural inhibition as well as a cognitive disruption in DBA mice, which should be taken into account when cognitively testing these animals.

Acknowledgements The skilled technical assistance of Ursula Habersetzer is gratefully acknowledged. This work was supported by the Volkswagen-Stiftung (Ohl: I/76268). 


\section{References}

Albrecht U, Oster H (2001) The circadian clock and behaviour. Behavioural Brain Research 125, $89-91$

Ammasari-Teule A, Tozzi A, Rossi-Arnaud C, Save E, Thinus-Blance C (1995) Reactions to spatial and nonspatial change in two inbred strains of mice, further evidence supporting the hippocampal dysfunction in the DBA/2 strain. Psychobiology 23, $284-9$

Barbini B, Bertelli S, Colombo C, Smeraldi E (1996) Sleep loss, a possible factor in augmenting manic episode. Psychiatry Research 65, 121-5

Becker A, Grecksch G (1996) Illumination has no effect on rats' behaviour in the elevated plus-maze. Physiology and Behaviour 59, 1175-7

Benstaali C, Mailloux A, Bogdan A, Auzéby A, Touitou Y (2001) Circadian rhythms of body temperature and motor activity in rodents, their relationships with the light-dark cycle. Life Sciences 68, 2645-56

Bertoglio LJ, Carobrez AP (2002) Behavioural profile of rats submitted to session 1 -session 2 in the elevated plus-maze during diurnal/nocturnal phases and under different illumination conditions. Behavioural Brain Research 132, 135-43

Cahib S, Puglisi-Allegra S, Ventura R (2002) The contribution of comparative studies in inbred strains of mice to the understanding of the hyperactive phenotype. Behavioural Brain Research 130, 103-9

Campuzano A, Cambras T, Vilaplana J, Canal MM, Carulla M, Diéz-Noguera A (1999) Period length of the light-dark cycle influences the growth rate in food intake in mice. Physiology and Behaviour 67, 791-7

Chaudhury D, Colwell CS (2002) Circadian modulation of learning and memory in fear-conditioned mice. Behavioural Brain Research 133, 95-108

Crawley JN, Belknap JK, Collins A, et al. (1997) Behavioural phenotypes of inbred mouse strains, implications and recommendations for molecular studies. Psychopharmacology 132, 107-24

Devan BD, Goad EH, Petri HL, et al. (2001) Circadian phase-shifted rats show normal acquisition but impaired long-term retention of place information in the water task. Neurobiology of Learning and Memory 75, 51-62

Gordon CJ, Becker P, Ali JS (1998) Behavioural thermoregulatory responses of single- and grouphoused mice. Physiology and Behaviour 65, 255-62

Griebel G, Sanger DJ, Perrault G (1997) Genetic differences in the mouse defence test battery. Aggressive Behaviour 23, 10-31

Hascoet M, Bourin M, Nic Dhonnchadha AB (2001) The mouse light-dark paradigm, a review. Progress in Neuropsychopharmacology and Biological Psychiatry 25, 141-66
Johns N, King SM (2001) Influence of circadian phase and test illumination on pre-clinical models of anxiety. Physiology and Behaviour 72, 99-106

Kamei Y, Urata J, Uchiyaya M, et al. (1998) Clinical characteristics of circadian rhythm sleep disorders. Psychiatry and Clinical Neuroscience 52, 234-5

Kelliher P, Connor TJ, Harkin A, Sanchez C, Kelly JP, Leonard BE (2000) Varying responses to the rat forced-swim test under diurnal and nocturnal conditions. Physiology and Behaviour 69, $531-9$

Kopp C (2001) Locomotor activity rhythm in inbred strains of mice, implications for behavioural studies. Behavioural Brain Research 125, 93-6

Kopp C, Vogel E, Rettori MC, Delagrange P, Guardiola-Lemaitre B, Misslin R (1998) Effects of a daylight cycle reversal on locomotor activity in several inbred strains of mice. Physiology and Behaviour 63, 577-85

Leamann TL (1999) Anxiety disorders. Mental Health 26, $197-210$

Mistlberger RE, Holmes MM (2001) Behavioural feedback regulation of circadian rhythm phase angle in light-dark entrained mice. American Journal of Physiology - Regulatory, Integrative and Comparative Physiology 297, 813-21

Nguyen PV, Abel T, Kandel ER, Bourtchouladze R (2000) Strain-dependent differences in LTP and hippocampus-dependent memory in inbred mice. Learning and Memory 7, 170-9

Ohl F, Oitzl MS, Fuchs E (1998) Assessing cognitive functions in tree shrews, visuo-spatial and spatial testing in the home cage. Journal of Neuroscience Methods 81, 5-40

Ohl F, Roedel A, Binder E, Holsboer F (2003) Impact of high and low anxiety on cognitive performance in a modified hole board test in C57BL/6 and DBA/2 mice. European Journal of Neuroscience $17,1-9$

Ohl F, Roedel A, Storch C, Holsboer F, Landgraf R (2002) Cognitive performance in rats differing in their inborn anxiety. Behavioural Neuroscience 116, 464-71

Ohl F, Sillaber I, Binder E, Keck ME, Holsboer F (2001a) Differential analysis of behaviour and diazepam-induced alterations in C57BL/6N and $\mathrm{BALB} / \mathrm{c}$ mice using the modified hole board test. Journal of Psychiatry Research 35, 147-54

Ohl F, Toschi N, Wigger A, Henninger MSH, Landgraf R (2001b) Dimensions of emotionality in a rat model of innate anxiety. Behavioural Neuroscience 115, 429-36

Parmigiani S, Palanza P, Rodger J, Ferrari PF (1999) Selection, evolution of behaviour and animal models in behavioural neuroscience. Neuroscience and Biobehavioural Reviews 23, 957-70

Ralph MR, Mrosovsky N (1992) Behavioural-inhibition of circadian responses to light. Journal of Biological Rhythms 7, 353-9 
Rodgers RJ, Haller J, Holmes A, Halasz J, Walton TJ, Brain PF (1999) Corticosterone response to the plus-maze, high correlation with risk-assessment in rats and mice. Physiology and Behaviour 68, 47-53

Ruiter M, La Fleur SE, van Heijningen C, van der Vliet J, Kalsbeek A, Buijs RM (2003) The daily rhythm in plasma glucagon concentrations in the rat is modulated by the biological clock and by feeding behaviour. Diabetes 52, 1709-15

Schwegler H, Crusio WE, Brust I (1990) Hippocampal mossy fibers and radial-maze learning in the mouse, a correlation with spatial working memory but with non-spatial reference memory. Neuroscience 34, 293-8

Stephan FK, Zucker I (1972) Circadian rhythms in drinking behaviour and locomotor activity of rats are eliminated by hypothalamic lesions. Proceedings of the National Academy of Sciences USA 69, 1583-6

Takahashi JS, Turek FW (2000) Locomotor response to an open field during C57BL/6J active and inactive phases, differences dependent on conditions of illumination. Physiology and Behaviour 69, 269-75

Thinus-Blanc C, Save E, Rossi-Arnaud C, Tozzi A, Ammassari-Teule M (1996) The differences shown by C57BL/6 and DBA/2 inbred mice in detecting spatial novelty are subserved by a different hippocampal and parietal cortex interplay. Behavioural Brain Research 80, 33-40
Trullas R, Skolnick P (1993) Differences in fear motivated behaviours among inbred mouse strains. Psychopharmacology 11, 323-31

Valentinuzzi VS, Buxton OM, Chang AM, et al. (2000) Locomotor response to an open field during C57BL/ $6 \mathrm{~J}$ active and inactive phases, differences dependent on conditions of illumination. Physiology and Behaviour 69, 269-75

Van den Pol AN, Cao V, Heller HG (1998) Circadian system of mice integrates brief light stimuli. American Journal of Physiology - Regulatory Integrative and Comparative Physiology 44, 654-7

Van der Hoofdakker RH, Beersma DGM (1988) On the contribution of sleep wake physiology to the explanation and the treatment of depression. Acta Psychiatrica Scandinavica 77, 53-71

Weinert D, Sturm J, Waterhouse J (2002) Different behaviour of the circadian rhythms of activity and body temperature during resynchronisation following an advance of the LD cycle. Biological Rhythm Research 33, 187-97

Weinert D, Waterhouse J (1998) Diurnally changing effects of locomotor activity on body temperature in laboratory mice. Physiology and Behaviour 63, $837-43$

Winocur G, Hasher L (1999) Aging and time-of-the day effects on cognition in rats. Behavioural Neuroscience 113, 991-7 